\title{
CUL4B promotes proliferation and inhibits apoptosis of human osteosarcoma cells
}

\author{
ZHI CHEN ${ }^{1 *}$, BAO-LIANG SHEN ${ }^{2 *}$, QING-GE FU ${ }^{3 *}$, FEI WANG $^{1}$, \\ YI-XING TANG ${ }^{1}$, CANG-LONG HOU ${ }^{1}$ and LI CHEN $^{1}$ \\ ${ }^{1}$ Department of Orthopedics, Changhai Hospital Affiliated to the Second Military Medical University, \\ Shanghai 200433; ${ }^{2}$ Department of Orthopedics, Shanghai Jiading Central Hospital, Shanghai 201800; \\ ${ }^{3}$ Department of Emergency and Trauma, Shanghai East Hospital, Tongji University \\ School of Medicine, Shanghai 200120, P.R. China
}

Received March 4, 2014; Accepted June 10, 2014

DOI: 10.3892/or.2014.3465

\begin{abstract}
Cullin 4B (CUL4B) is a component of the Cullin4B-Ring E3 ligase complex (CRL4B) that functions in proteolysis and is implicated in tumorigenesis. Here, we report that $C U L 4 B$ is associated with tumorigenesis by promoting proliferation and inhibiting apoptosis of human osteosarcoma cells. We performed RNA interference (RNAi) with a lentiviral vector system to silence the $C U L 4 B$ gene using osteosarcoma SAOS-2 cells. The negative control included the normal target cells infected with the negative control virus whereas the knockdown cells included the normal target cells transfected with the RNAi target virus. We assessed the inhibition resulting from the decreased expression of the $C U L 4 B$ gene on the proliferation rate of SAOS-2 cells, and also evaluated the cell cycle distribution, apoptosis and clonability. Compared with the negative control, the $C U L 4 B$ gene expression was significantly inhibited in the SAOS-2 cells at the mRNA and protein levels in the knockdown group $(\mathrm{P}<0.01)$. Furthermore, in the knockdown group, the cell proliferation rate and clonability were also significantly inhibited $(\mathrm{P}<0.01)$. The apoptosis rate increased significantly $(\mathrm{P}<0.05)$. A significant decrease in the number of cells in the G1 phase $(\mathrm{P}<0.01)$ and significant increases in the $\mathrm{S}(\mathrm{P}<0.01)$ and $\mathrm{G} 2$ phases $(\mathrm{P}<0.05)$ were observed. The silencing of $C U L 4 B$ gene expression can effectively inhibit osteosarcoma cell proliferation and induce apoptosis. These findings may provide a novel biomarker for the treatment of osteosarcoma.
\end{abstract}

Correspondence to: Dr Li Chen, Department of Hematology, Changhai Hospital Affiliated to the Second Military Medical University, 168 Changhai Road, Shanghai 200433, P.R. China E-mail: chenli37t@gmail.com

${ }^{*}$ Contributed equally

Key words: CUL4B, osteosarcoma cells, proliferation, apoptosis

\section{Introduction}

Osteosarcoma is a primary bone malignancy with high rates of metastasis, mortality and disability, and usually occurs in children and adolescents (1). The most common treatments for osteosarcoma are surgery, chemotherapy and biotherapy. However, the prognosis of osteosarcoma is still poor because of the high degree of malignancy, rapid disease progression and early metastasis (2).

Gene amplification can be defined as increased copy numbers of certain regions of the genome. Gene amplification often results in the upregulation of gene expression by increasing the number of templates available for transcription. Gene amplification is commonly found in tumor cells and is considered an important mechanism whereby tumor cells gain increased levels of expression of critical genes. Thus, identification and characterization of these genes should provide important insights into the pathobiology of cancer.

Culllin $4 \mathrm{~B}(C U L 4 B)$ is a component of the Cullin4B-Ring E3 ligase complex $(C R L 4 B)$ that functions in proteolysis. It has been implicated in tumorigenesis since the core components participate in a broad variety of physiologically and developmentally controlled processes such as cell progression, replication and DNA damage response (3). In mammals, there are two Cullin 4 members, $C U L 4 A$ and $C U L 4 B$, which share $82 \%$ sequence identity. Although $\mathrm{Cul} 4 \mathrm{a}$-null mice do not exhibit evident developmental defects (4-6), CUL4A has been shown to target p53 and several cyclin-dependent kinase inhibitors, such as p21 and p27 for proteolysis in cultured cells (7-9) and was found to be highly expressed in breast and hepatocellular carcinomas $(10,11)$. Loss-of-function mutation in the X-linked $C U L 4 B$, in contrast, causes mental retardation, short state, absence of speech and other phenotypes in humans $(12,13)$, and $C u l 4 B$ knockout mice are embryonically lethal, indicating a unique function of $C U L 4 B$ that cannot be compensated by $C U L 4 A$. Recently, Yang et al (14) showed that depletion of CUL4B resulted in loss of not only H2AK119 mono-ubiquitination but also $\mathrm{H} 3 \mathrm{~K} 9$ tri-methylation and DNA methylation, leading to depression of a collection of genes, including the tumor-suppressor IGFBP3. Further experiments revealed that CUL4B promoted cell proliferation and invasion, 
which are consistent with a tumorigenic phenotype, at least partially by repressing IGFBP3. Further findings revealed that the expression of CUL4B was markedly upregulated in samples of human cervical carcinoma and was negatively correlated with the expression of IGFBP3.

Although experiments have shown that $C U L B$ possesses an intrinsic transcription repressive activity, $(3,15)$ the exact role of CULB in the process and progression of osteosarcoma is still poorly understood. Here, we established an osteosarcoma cell model in vitro, and transfected osteosarcoma SAOS-2 cells using CUL4B RNAi and assessed the apoptosis of SAOS-2 cells. We demonstrated that CUL4B promotes cell proliferation and inhibits the apoptosis of osteosarcoma cells. These results add to the understanding of the role of CUL4B in the proliferation and apoptosis of osteosarcoma cells and its molecular mechanisms.

\section{Materials and methods}

Cell line and culture conditions. Human osteosarcoma cell line SAOS-2 was purchased from the Shanghai Institute of the Chinese Academy of Sciences (Shanghai, China) and grown in Dulbecco's modified Eagle's medium (DMEM) with 10\% (v/v) fetal calf serum, streptomycin $(100 \mathrm{U} / \mathrm{ml})$ and penicillin (100 U/ml). DMEM, fetal bovine serum (FBS) and Dimethyl sulfoxide (DMSO) were purchased from Gibco Biotechnology (Gibco-BRL, MD, USA). Cultures were maintained at $37^{\circ} \mathrm{C}$ in an incubator with a humidified atmosphere of $5 \% \mathrm{CO}_{2}$.

Reverse transcription-polymerase chain reaction (RT-PCR) analysis of CUL $4 B$ gene expression in tumor cells. Total-RNA was extracted under RNase-free conditions (carried out according to the operation manual for TRIzol; Invitrogen Corp., Carlsbad, CA, USA) with glyceraldehyde-3-phosphate dehydrogenase (GAPDH) as an internal reference. Primer design was as follows: the CUL4B upstream sequence was 5'-GGGAAAGGAATGGTGAA-3'; and the downstream sequence was 5'-TGCATAGAGCCGGTTAG-3'. The GAPDH upstream sequence was 5'-TGACTTCAACAGCGACAC CCA-3'; and its downstream sequence was 5'-CACCCTGTT GCTGTAGCCAAA-3'. The Access RT-PCR kit was used to perform single-step reverse transcription and PCR amplification. An aliquot of $5 \mu \mathrm{l}$ of the amplified products was subjected to electrophoresis on $2 \%$ agarose gel. The gels were examined under a UV lamp.

Construct design: lentiviral-mediated small interfering RNA delivery system. We targeted the gene of interest by designing small interfering RNAs (siRNAs) using the design software developed by Ambion Corp. (Naugatuck, CT, USA) to select the best parameters for the RNA interference target. We determined the effective target sequence: PSCSI2891, AGCAGTGGAAGCTATTCAGAA (CUL4B mRNA). We designed the DNA oligonucleotides of siRNAs (Shanghai Genechem Co., Ltd.): PSCSI2891-1, 5'-CcggAGCAGTGGA AGCTATTCAGAATTCAAGAGATTCTGAATAGCTTCCA CTGCTTTTTTg-3' and PSCSI2891-2, 5'-aattcaaaaaAGCAG TGGAAGCTATTCAGAATCTCTTGAATTCTGAATAGCT TCCACTGCT-3'. After annealing, the double-stranded DNA was digested with Agel and EcoRI (New England Biolabs) to linearize the pGCSIL-GFP vector. We modified the doublestranded DNA after annealing and linked it with the pGCSIL-GFP vector following the double digestion. We used calcium chloride to prepare competent cells of Escherichia coli afresh and cultured it at $37^{\circ} \mathrm{C}$ for $16 \mathrm{~h}$. We used computeraided high-throughput cloning of bacteria in liquid medium for sequencing (Shanghai Genechem Co., Ltd.).

Preparation and grouping of cells. The human osteosarcoma cell line SAOS-2 was cultured in the RPMI-1640 culture solution with fetal calf serum (volume fraction 10\%) and incubated with $5 \% \mathrm{CO}_{2}$ at $37^{\circ} \mathrm{C}$. The cells that remained in the logarithmic phase were divided into two groups: i) negative control, in which the normal target cells were infected withthe negative control virus and ii) knockdown, in which the normal target cells were infected with the RNAi target virus.

Real-time PCR and western blotting to test knockdown efficiency. The human osteosarcoma SAOS-2 cells grew well on the day prior to viral introduction was recovered. The cell suspension was incubated with $5 \% \mathrm{CO}_{2}$ at $37^{\circ} \mathrm{C}$. When the degree of cell fusion reached $30 \%$, adequate viral load was introduced in the different groups, to a multiplicity of infection (MOI) of 100. Following incubation for three days, the expression level of GFP was observed under a fluorescence microscope. The culture was continued if the efficiency of infection exceeded 50\%. After incubation for five days, the cells were collected; the mRNA expression of the gene of interest was analyzed using real-time PCR for RNA interference. The upstream primer sequence for the $C U L 4 B$ gene was 5'-GGGAAAGGAATGGTGAA-3' while the downstream primer sequence was 5'-TGCATAGAGCCGGTTAG-3'. The cell culture solution was aspirated and the cells were washed twice in phosphate-buffered saline (PBS). An adequate amount of pre-cooled 2X lysis buffer was added. After deplating, the cells were transferred to the tube and then lysed on ice for 10-15 min. The protein concentration was determined and adjusted to $2 \mu \mathrm{g} / \mu \mathrm{l}$. Next, $2 \mathrm{X}$ loading buffer was added to each sample and subsequently boiled for 5-10 min. Forty micrograms of total protein was loaded into each well containing $10 \%$ SDS-polyacrylamide gel, subjected to electrophoresis at $30 \mathrm{~mA}$ for $2 \mathrm{~min}$, and then transferred to a polyvinylidene fluoride (PVDF) membrane at $400 \mathrm{~mA}$ for $2 \mathrm{~h}$. The membrane was blocked with 5\% milk in Tris-buffered saline (TBS) for $1 \mathrm{~h}$ at room temperature. The primary antibody was added and incubated with the membrane for $2 \mathrm{~h}$ at room temperature, and then washed three times with TBS/Tween-20. A secondary antibody was added to the membrane and incubated at room temperature with gentle agitation. Two hours later, the membrane was washed three times with TBS/Tween-20 for $10 \mathrm{~min}$ per wash. The bands were visualized using an enhanced chemilluminescence (ECL) kit followed by exposure to X-ray film.

Cellomics to test inhibition of osteosarcoma cell proliferation following downregulation of the CUL4B gene. After trypsinization, the cell suspension was resuspended in complete medium and density was adjusted to $2 \times 10^{4} / \mathrm{ml}$. We used the blood counting chamber to count the cells, laying them at 2,000 cells/well. Each group comprised three to five compound 
perforations. Each perforation was filled with $100 \mu$, and the same quantity of cells. The cells were incubated with $5 \%$ $\mathrm{CO}_{2}$ and cultured at $37^{\circ} \mathrm{C}$. Starting the next day, the plates were tested and read once a day with Cellomics for five days. After adjusting the input parameters of Cellomics ArrayScan (Thermo Fisher Scientific Inc., Waltham, MA, USA), the quantity of cells with green fluorescence was accurately calculated while scanning the perforations in the plates. The data were collected and analyzed to create a proliferation curve for the five days.

Fluorescence-activated cell sorting (FACS) to assess osteosarcoma cell cycle distribution following CUL4B gene silencing. The cell culture supernatant was aspirated when the coverage rate for the $6-\mathrm{cm}$ dish cells in the experimental group increased to $80 \%$, ensuring that the cells did not enter the plateau phase. The cells were washed once with D-Hank's solution and subjected to trypsinization. The complete medium was removed and the cells were collected in a 5-ml centrifuge tube. We set three compound perforations in each group and performed timed cycle tests ensuring an adequate number of cells for computerized analysis, with at least 1,000,000 each time. We used PBS ( $\mathrm{pH}, 7.2-7.4)$ that was pre-cooled at $4^{\circ} \mathrm{C}$ to wash and precipitate cells once. The cells were collected after centrifugation at $1,500 \mathrm{rpm}$ for $5 \mathrm{~min}$. The cells were fixed with $70 \%$ ethanol, which was pre-cooled to $4^{\circ} \mathrm{C}$, for at least $1 \mathrm{~h}$. The stationary liquid was abandoned by centrifugation at 1,500 rpm for $5 \mathrm{~min}$. We used PBS to wash and precipitate cells once. Adequate quantity of cell staining fluid $(1-1.5 \mathrm{ml})$ was added for suspension, based on the volume of cells, ensuring that the pass rate of cells reached $200-350 \mathrm{cell} / \mathrm{sec}$ for computer analysis. We used a 300-mesh screen cloth to filter within the tube while streaming onto the computer.

Fluorescence-activated cell sorting (FACS) analysis of apoptosis following downregulation of the CUL $4 B$ gene. We used D-Hank's solution to wash cells once after collecting the culture supernatant from each experimental group following transfer into the 5-ml centrifuge tube. We used pancreatic enzymes to digest the cells. The culture supernatants were then abandoned, and cells were collected into one 5-ml centrifuge tube, with three compound perforations in each group. The supernatants were aspirated after centrifugation at 1,500 rpm for $5 \mathrm{~min}$. We used PBS to wash and precipitate the cells once and then cells were collected after centrifugation at $1,500 \mathrm{rpm}$ for $5 \mathrm{~min}$. A $1 \mathrm{X}$ binding buffer was then used to wash and precipitate cells once, and centrifuged at 1,500 rpm for $5 \mathrm{~min}$. The collected cells were resuspended using $1 \mathrm{ml} 1 \mathrm{X}$ staining buffer; the volume of staining buffer solution was determined according to the precipitation capacity of the cells, adjusting the final density of the cell suspension to $1 \times 10^{6}-1 \times 10^{7}$ cells $/ \mathrm{ml}$. We took a $100-\mu 1$ cell suspension $\left(1 \times 10^{5}-1 \times 10^{6}\right.$ cells $)$ and added $5 \mu \mathrm{l}$ Annexin V-APC for dyeing. The mixture was placed in a dark place at room temperature for 10-15 min, and then transferred to the tube for streaming onto the computer for further analysis.

Inhibition of clonability of the osteosarcoma cells following downregulation of the CUL $4 B$ gene. We digested cells that remained in the logarithmic phase in each experimental group, with pancreatic enzymes. The cells were resuspended

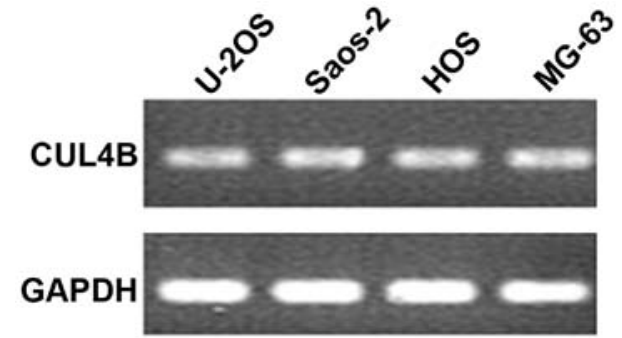

Figure 1. $C U L 4 B$ gene mRNA expression in SAOS-2 cells.

in complete medium. Using a blood counting chamber, we inoculated the 96-well culture plate at the rate of 500 cells per perforation in each experimental group. We set three compound perforations in each experimental group, transferred the cells that were already inoculated into the incubator and they were cultured for three days or when the number of cells in most single clones exceeded five. During the process, the solution was changed and cells were monitored every three days. Using Cellomics ArrayScan, we scanned and photographed the perforations, analyzed the number and size of the clones within the perforations and the number of cells in each clone.

Statistical analysis. All data are expressed as mean \pm standard deviation (SD). We used the statistical software SPSS 12.0 to perform the relevant analysis. The significance level of statistics was set at $\mathrm{P}<0.05$.

\section{Results}

Expression of the CULAB gene in SAOS-2 cells, preparation of RNA-interfering lentivirus vector and assessment of knockdown efficiency. The results of the semi-quantitative PCR, which set GAPDH as an internal reference, showed that the $C U L 4 B$ gene was abundantly expressed in the osteosarcoma SAOS-2 cells (Fig. 1). The length of the PCR fragment in the positive clone that annealed with the fragment of vshRNA was $343 \mathrm{bp}$. After transfection with the siRNA lentivirus for three days, GFP expression was observed under fluorescence microscopy (Fig. 2). After five days, it was found that the mRNA expression level of the CUL $4 B$ gene in the SAOS-2 cells of the knockdown group was inhibited, which was significantly different compared with the negative control group. The western blotting results confirmed inhibition of the protein level by the silencing of the $C U L 4 B$ gene in SAOS-2 cells. The RNA-interfering lentivirus of the $C U L 4 B$ gene construct effectively inhibited the expression of the $C U L 4 B$ gene and several targets following gene silencing (Fig. 3).

Cellomic analysis of inhibition of the proliferation of osteosarcoma cells following downregulation of the CUL4B gene. After transfection with the siRNA lentivirus, the proliferation rate of SAOS-2 cells in the knockdown group was inhibited together with the negative control from the third day $(\mathrm{P}<0.01)$. The results suggested that downregulation of the CUL4B gene inhibited the proliferation of SAOS-2 (Fig. 4).

FACS analysis of cell cycle distribution following CUL4B gene silencing. Following transfection with the siRNA lentivirus, 

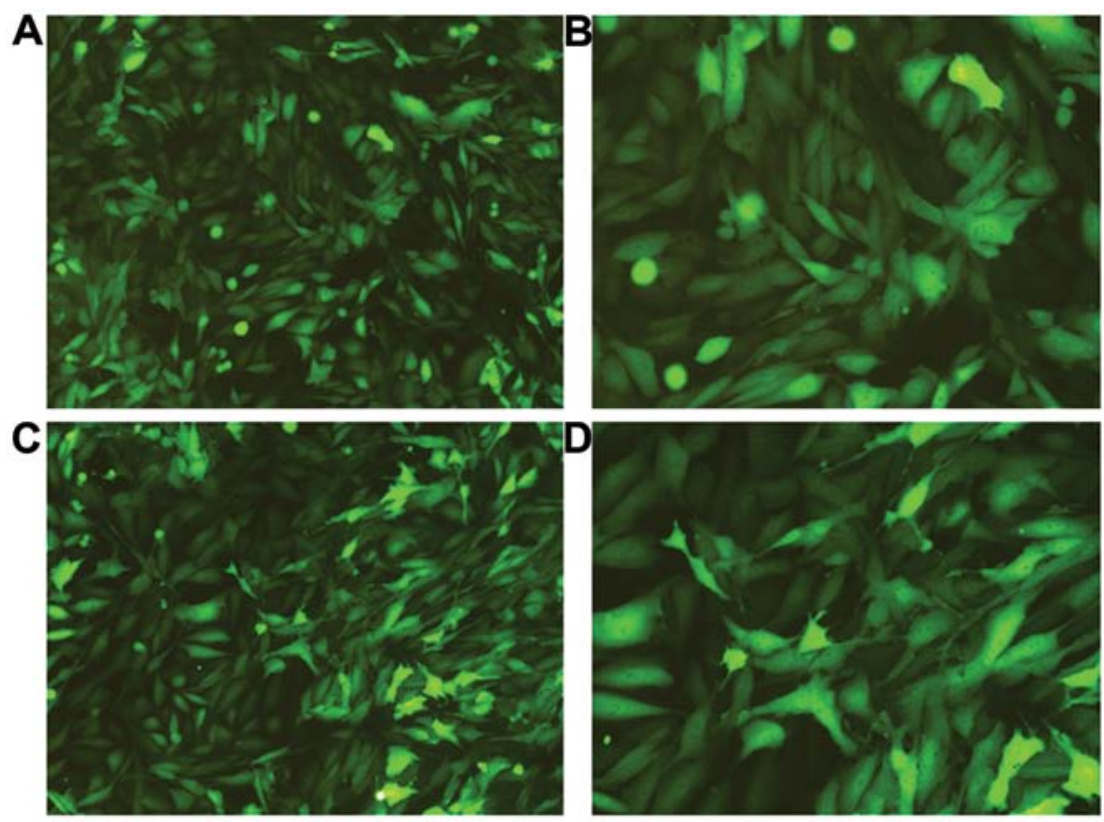

Figure 2. GFP expression in SAOS-2 cells as detected by fluorescence microscope. (A) Negative-control; magnification x100. (B) Negative-control; magnification $\mathrm{x} 200$. (C) $C U L 4 B$-siRNA; magnification $\times 100$. (D) CUL4B-siRNA; magnification x200.

A

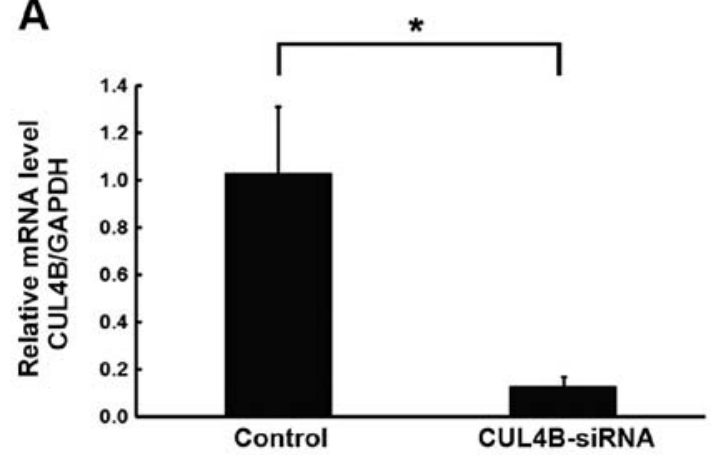

B

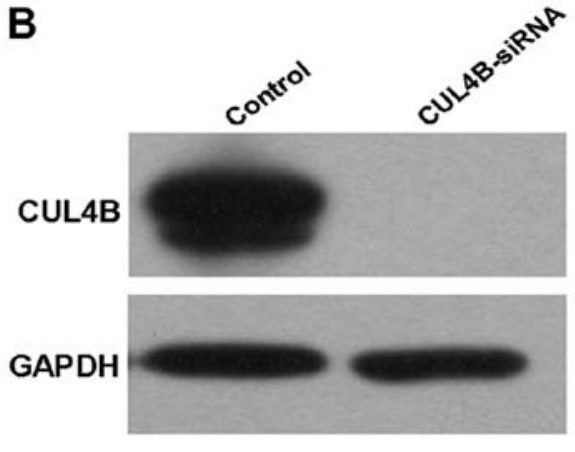

Figure 3. $C U L 4 B$ gene expression in SAOS-2 cells following knockdown of the $C U L 4 B$ gene. (A) In the knockdown group, the mRNA expression of the $C U L 4 B$ gene in the SAOS-2 cells was decreased ( ${ }^{* *} \mathrm{P}<0.01$, CUL4B-siRNA vs. negative-control). (B) In the knockdown group, the protein expression of $C U L 4 B$ in the SAOS-2 cells was decreased.

the percentage of SW1116 cells in the G1 phase was significantly decreased $(\mathrm{P}<0.01)$, while cells in the $\mathrm{S}(\mathrm{P}<0.01)$ and $\mathrm{G} 2$ phases increased significantly $(\mathrm{P}<0.05)$ in the knockdown group compared with the negative control group. This indicates that downregulation of the CUL4B gene was apparently related to regular distribution of the SW1116 cells (Fig. 5).

FACS analysis of apoptosis following CUL4B gene silencing. Transfection by siRNA lentivirus increased the rate of apoptosis significantly $(\mathrm{P}<0.05)$ in the knockdown group compared with the negative control group, suggesting that the CUL4B gene silencing stimulated the apoptosis of SAOS-2 cells (Figs. 6 and 7).

Analysis of inhibition of clonability following CUL4B gene silencing. We noticed a decrease in the number of SW1116 cell colonies in the knockdown group following interference by siRNA lentivirus. Furthermore, the number of cells in the colonies that had already formed decreased, which was signifi- cantly different from the negative control group $(\mathrm{P}<0.01)$. The results suggest that downregulation of the CUL4B gene largely inhibited the clonability of the SAOS-2 cells (Fig. 8).

\section{Discussion}

In the present study, using RNA interference with a lentiviral vector containing the $C U L 4 B$ gene, we knocked down the expression of the $C U L 4 B$ gene in osteosarcoma SAOS-2 cells. Our results demonstrated that the knockdown of $C U L 4 B$ inhibited osteosarcoma SAOS-2 cell proliferation and clonability and significantly induced apoptosis. Our findings suggest that silencing of CUL4B gene may be used in the treatment of human osteosarcoma.

Cancer cells commonly develop mechanisms by which they resist cell death either through disruption of apoptotic processes or activation of survival signals. Within a growing tumor mass, the sequential acquisition of a number of genetic and epigenetic alterations during tumor progression also enable 
A
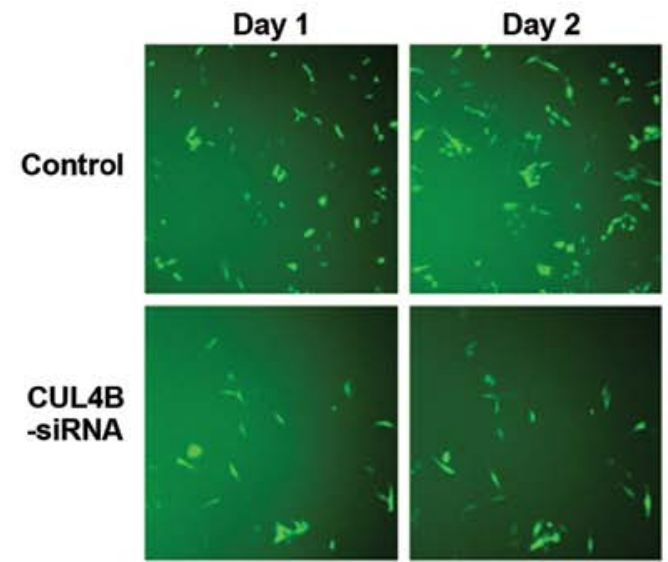
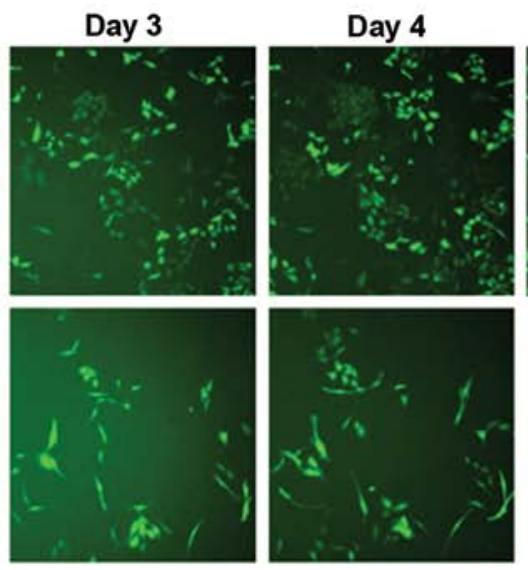
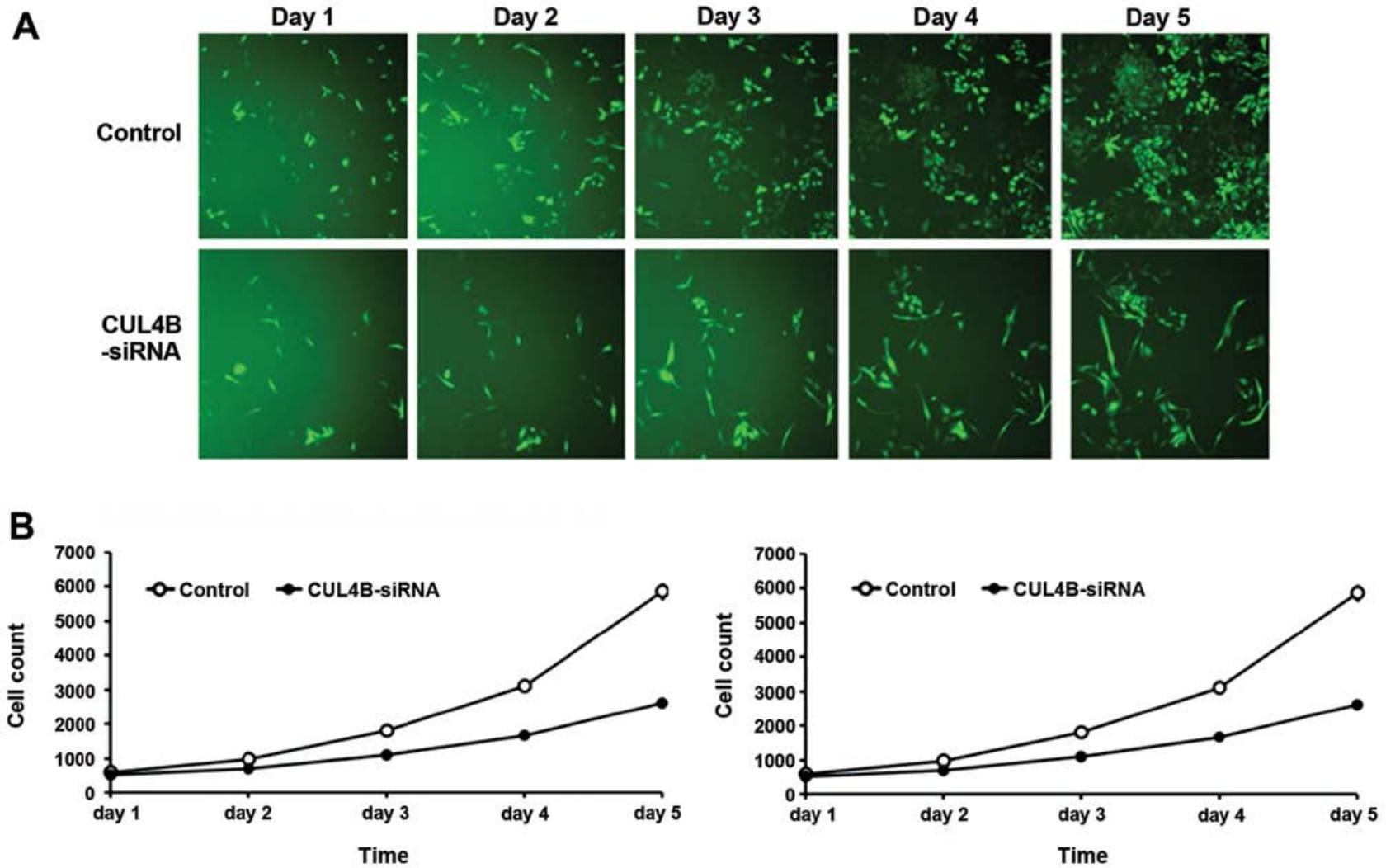

Figure 4. Downregulation of the CUL $4 B$ gene inhibits proliferation of SAOS-2 cells. (A) Proliferation of SAOS-2 cells in the knockdown group was inhibited when compared with the negative-control group (** $\mathrm{P}<0.01$, CUL4B-siRNA vs. negative-control). (B) Proliferation rate of SAOS-2 cells in the knockdown group was inhibited when compared with the negative-control group ( ${ }^{* *} \mathrm{P}<0.01$, CUL4B-siRNA vs. negative-control).
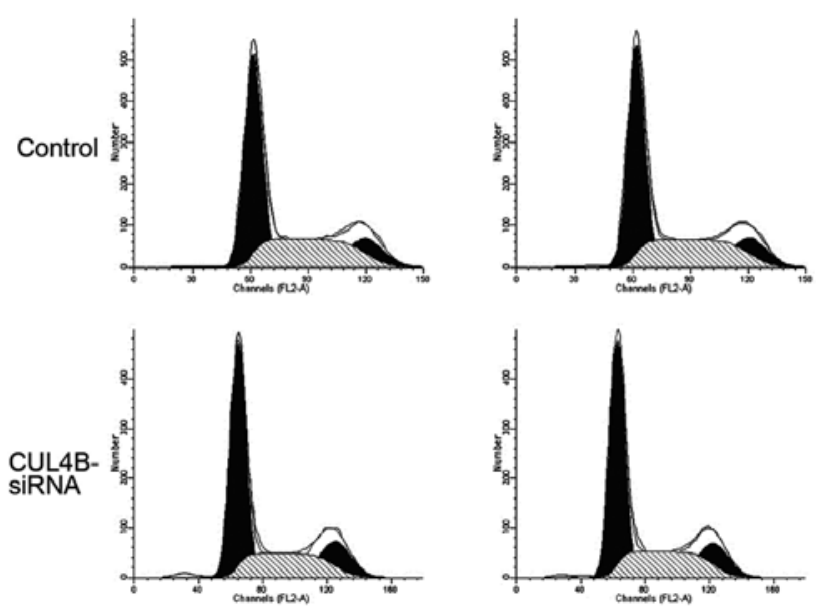
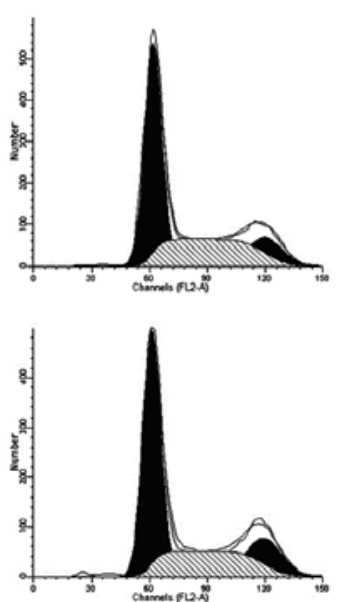

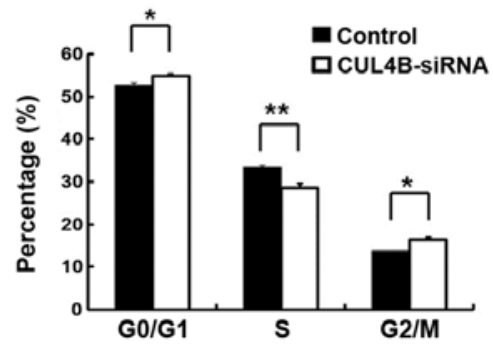

Figure 5. Changes in the cell cycle distribution of SAOS-2 cells in the knockdown group ${ }^{* * *} \mathrm{P}<0.01, \mathrm{CUL} 4 \mathrm{~B}-\mathrm{siRNA}$ vs. negative-control; ${ }^{*} \mathrm{P}<0.05, \mathrm{CUL} 4 \mathrm{~B}-$ siRNA vs. negative-control).

cancer cells to gain the ability to escape apoptosis, induce angiogenesis and metastasize to distant organs. Notably, CUL4B carries a nuclear localization signal in its $\mathrm{N}$ terminus and is also localized in the nucleus $(15,16)$, suggesting that CUL4B might be involved in nuclear-based functions. Recently, one study indicated that CUL4B is a transcriptional corepressor that regulates transcription by recruiting PRC2. It was demonstrated that CUL4B functions as a transcription corepressor and a potential oncogene, supporting the use of CUL4B as a target for cancer therapy (17).
Although the mechanism of CUL4B in cancer development and progression remains unclear, studies have shown that CUL4B may function together with other proteins, such as cyclin D1 and p53 $(7,18)$. The cyclin D1 level was low in quiescent cells, and it increased as cells progressed into the G1 phase and served as a control protein (19). P53 also known as cellular tumor antigen p53 or tumor-suppressor p53 is a protein that in humans is encoded by the TP53 gene. The p53 protein is crucial in multicellular organisms, where it regulates the cell cycle and, thus, functions as a tumor suppressor, preventing cancer (20). 

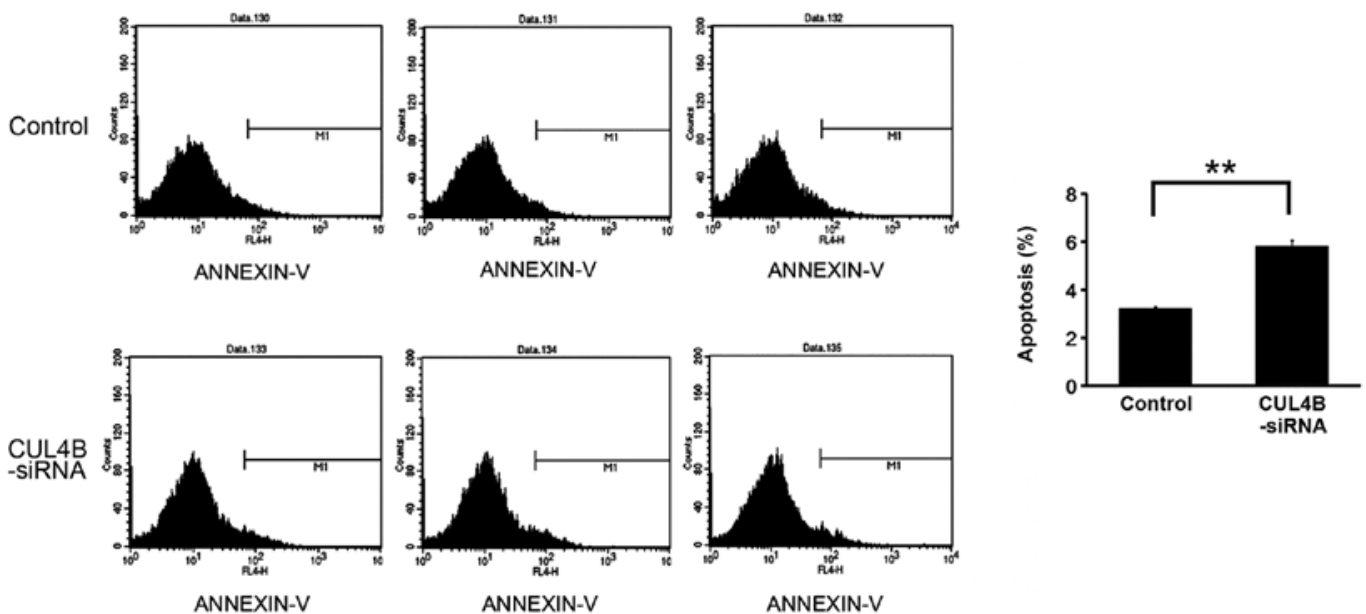

Figure 6. Downregulation of the CUL4B gene stimulates apoptosis of SAOS-2 cells (Peak diagram). ("P<0.05, CUL4B-siRNA vs. negative-control).
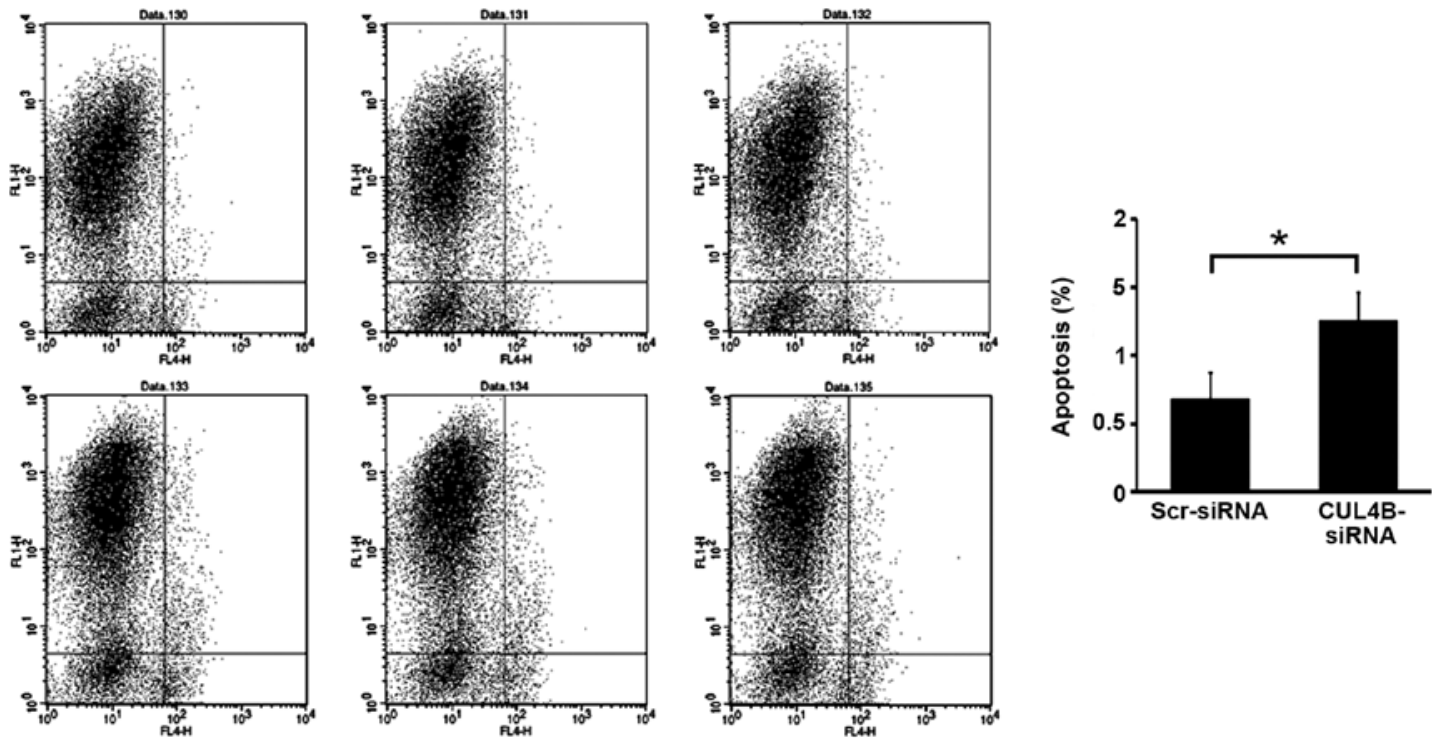

Figure 7. Downregulation of the CUL4B gene promotes apoptosis of SAOS-2 cells (Scatter diagram). (" $\mathrm{P}<0.05, \mathrm{CUL} 4 \mathrm{~B}$-siRNA vs. negative-control).
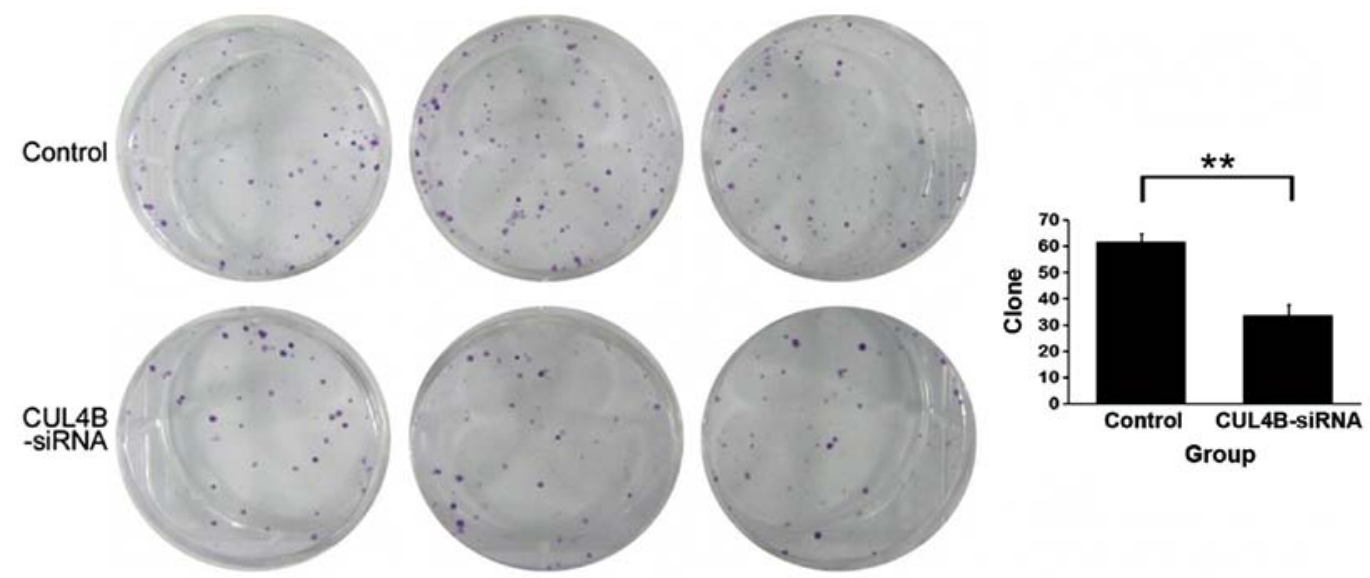

Figure 8. Compared with the negative-control group, the clonability of SAOS-2 cells in the knockdown group was significantly inhibited ("P $<0.01$, CUL4B-siRNA vs. negative-control).

CUL4-mediated ubiquitination, which is involved in other types of histone modifications and epigenetic mechanisms, such as heterochromatin formation, parental imprinting or
X-chromosome inactivation (21-23), may play an important role in cancer development. CUL4B expression was found to be significantly higher in tumor samples compared to 
adjacent normal tissue, and the level of CUL4B expression was negatively correlated with the level of IGFBP3 expression. Moreover, IGFBP3 is induced by wild-type p53 (24) and enhances the p53-dependent apoptotic response of tumor cells to DNA damage (25). Aberrant promoter methylation of IGFBP3 at the p53 regulatory element causes gene silencing resistant to p53 (26). Interestingly, it has been reported that CRL4A can degrade p53 to promote cell cycle progression and immortalization $(7,27)$. Thus, it is reasonable to speculate that CRL4 negatively controls the p53-IGFBP3 axis. Collectively, in addition to the hypothesis that CRL4B promotes tumorigenesis through degradation of several cyclin-dependent kinase inhibitors $(8,9)$ and coordinates with PRC2 in H3K27me3-mediated transcriptional silencing (17), recent research has also shown that CUL4B controls DNA methylation-based transcriptional repression adding a new element to the understanding of the oncogenic potential of CRL4B.

Progression of human cancers is known to involve multiple genetic changes that lead to upregulation or downregulation of expression of critical genes (28). Gene amplification is a common mechanism whereby tumor cells increase expression of genes that are critical for malignancy. However, not all genes present in multiple copies are necessarily relevant to malignancy. Amplicons may contain irrelevant genes that are present by virtue of their physical proximity to target genes. In this study, we investigated the biological function of the $C U L 4 B$ gene in tumorigenesis and proposed the possible mechanism based on previous research. We provide a new prospective for the role of CUL4B in tumorigenesis which warrants further research.

In summary, in our study, using RNA interference with a lentiviral vector containing the $C U L 4 B$ gene, we knocked down the expression of the $C U L 4 B$ gene in osteosarcoma SAOS-2 cells. Our results demonstrated that the knockdown of $C U L 4 B$ can inhibit osteosarcoma SAOS-2 cell proliferation and clonability and significantly induce apoptosis. Our findings suggest that silencing of the CUL4B gene may be valuable for the treatment of human osteosarcoma.

\section{Acknowledgements}

This study was supported by grants from The National Natural Science Foundation of China General Program (no. 120038), National Key Clinical Specialty Construction Project of China, Shanghai Municipality Key Clinical Specialty (ZK2012A28) and Sailing East Development Program of Shanghai East Hospital.

\section{References}

1. Ferrari S and Palmerini E: Adjuvant and neoadjuvant combination chemotherapy for osteogenic sarcoma. Curr Opin Oncol 19: 341-346, 2007.

2. Sasaki N, Toda T, Kaneko T, Baba N and Matsuo M: Protective effects of flavonoids on the cytotoxicity of linoleic acid hydroperoxide toward rat pheochromocytoma PC12 cells. Chem Biol Interact 145: 101-116, 2003.

3. Jackson S and Xiong Y: CRL4s: the CUL4-RING E3 ubiquitin ligases. Trends Biochem Sci 34: 562-570, 2009.

4. Kopanja D, Roy N, Stoyanova T, Hess RA, Bagchi S and Raychaudhuri P: Cul4A is essential for spermatogenesis and male fertility. Dev Biol 352: 278-287, 2011.

5. Liu L, Lee S, Zhang J, et al: CUL4A abrogation augments DNA damage response and protection against skin carcinogenesis. Mol Cell 34: 451-460, 2009.
6. Yin Y, Lin C, Kim ST, et al: The E3 ubiquitin ligase Cullin 4A regulates meiotic progression in mouse spermatogenesis. Dev Biol 356: 51-62, 2011.

7. Banks D, Wu M, Higa LA, et al: L2DTL/CDT2 and PCNA interact with $\mathrm{p} 53$ and regulate $\mathrm{p} 53$ polyubiquitination and protein stability through MDM2 and CUL4A/DDB1 complexes. Cell Cycle 5: 1719-1729, 2006.

8. Higa LA, Yang X, Zheng J, et al: Involvement of CUL4 ubiquitin E3 ligases in regulating CDK inhibitors Dacapo/p27Kip1 and cyclin E degradation. Cell Cycle 5: 71-77, 2006.

9. Nishitani H, Shiomi Y, Iida H, Michishita M, Takami T and Tsurimoto T: CDK inhibitor $\mathrm{p} 21$ is degraded by a proliferating cell nuclear antigen-coupled Cul4-DDB1Cdt2 pathway during S phase and after UV irradiation. J Biol Chem 283: 29045-29052, 2008.

10. Schindl M, Gnant M, Schoppmann SF, Horvat R and Birner P: Overexpression of the human homologue for Caenorhabditis elegans cul-4 gene is associated with poor outcome in nodenegative breast cancer. Anticancer Res 27: 949-952, 2007.

11. Singhal S, Amin K, Kruklitis R, et al: Alterations in cell cycle genes in early stage lung adenocarcinoma identified by expression profiling. Cancer Biol Ther 2: 291-298, 2003.

12. Tarpey PS, Raymond FL, O'Meara S, et al: Mutations in CUL4B, which encodes a ubiquitin E3 ligase subunit, cause an X-linked mental retardation syndrome associated with aggressive outbursts, seizures, relative macrocephaly, central obesity, hypogonadism, pes cavus, and tremor. Am J Hum Genet 80: 345-352, 2007.

13. Zou Y, Liu Q, Chen B, et al: Mutation in CUL4B, which encodes a member of cullin-RING ubiquitin ligase complex, causes X-linked mental retardation. Am J Hum Genet 80: 561-566, 2007.

14. Yang Y, Liu R, Qiu R, et al: CRL4B promotes tumorigenesis by coordinating with SUV39H1/HP1/DNMT3A in DNA methylation-based epigenetic silencing. Oncogene: Dec 2, 2013 (Epub ahead of print). doi: 1038/onc.2013.522.

15. Nakagawa $T$ and Xiong $Y$ : X-linked mental retardation gene CUL4B targets ubiquitylation of H3K4 methyltransferase component WDR5 and regulates neuronal gene expression. Mol Cell 43: 381-391, 2011.

16. Zou Y, Mi J, Cui J, et al: Characterization of nuclear localization signal in the $\mathrm{N}$ terminus of CUL4B and its essential role in cyclin E degradation and cell cycle progression. J Biol Chem 284: 33320-33332, 2009.

17. Hu H, Yang Y, Ji Q, et al: CRL4B catalyzes H2AK119 monoubiquitination and coordinates with PRC2 to promote tumorigenesis. Cancer Cell 22: 781-795, 2012.

18. Aggarwal P, Lessie MD, Lin DI, et al: Nuclear accumulation of cyclin D1 during S phase inhibits Cul4-dependent Cdt1 proteolysis and triggers p53-dependent DNA rereplication. Genes Dev 21: 2908-2922, 2007.

19. Koepp DM, Harper JW and Elledge SJ: How the cyclin became a cyclin: regulated proteolysis in the cell cycle. Cell 97: 431-434, 1999.

20. Levine AJ, Momand $\mathrm{J}$ and Finlay CA: The $\mathrm{p} 53$ tumour suppressor gene. Nature 351: 453-456, 1991.

21. Jia S, Kobayashi R and Grewal SI: Ubiquitin ligase component Cul4 associates with $\mathrm{Clr} 4$ histone methyltransferase to assemble heterochromatin. Nat Cell Biol 7: 1007-1013, 2005.

22. Horn PJ, Bastie JN and Peterson CL: A Rik1-associated, cullin-dependent E3 ubiquitin ligase is essential for heterochromatin formation. Genes Dev 19: 1705-1714, 2005.

23. Dumbliauskas E, Lechner E, Jaciubek M, et al: The Arabidopsis CUL4-DDB1 complex interacts with MSI1 and is required to maintain MEDEA parental imprinting. EMBO J 30: 731-743, 2011.

24. Buckbinder L, Talbott R, Velasco-Miguel S, et al: Induction of the growth inhibitor IGF-binding protein 3 by p53. Nature 377: 646-649, 1995

25. Williams AC, Collard TJ, Perks CM, et al: Increased p53-dependent apoptosis by the insulin-like growth factor binding protein IGFBP-3 in human colonic adenoma-derived cells. Cancer Res 60: 22-27, 2000.

26. Hanafusa T, Shinji T, Shiraha H, et al: Functional promoter upstream p53 regulatory sequence of IGFBP 3 that is silenced by tumor specific methylation. BMC Cancer 5: 9, 2005.

27. Nag A, Bagchi S and Raychaudhuri P: Cul4A physically associates with MDM2 and participates in the proteolysis of p53. Cancer Res 64: 8152-8155, 2004.

28. Dairkee SH and Smith HS: Genetic analysis of breast cancer progression. J Mammary Gland Biol Neoplasia 1: 139-151, 1996. 